Proceedings

of the

Combustion

Institute

\title{
Problem adapted reduced models based on Reaction-Diffusion Manifolds (REDIMs)
}

\author{
V. Bykov*, U. Maas \\ Institut für Technische Thermodynamik, Karlsruhe University, Kaiserstraße 12, D-76128 Karlsruhe, Germany
}

\begin{abstract}
In this work a novel modification of the REDIM method is presented. The method follows the main concept of decomposition of time scales. It is based on the assumption of existence of invariant slow manifolds in the thermo-chemical composition space (state space) of a reacting flow. A central point of the current modification is its capability to include both transport and thermo-chemical processes and their coupling into the definition of the reduced model. This feature makes the method more problem oriented, and more accurate in predicting the detailed system dynamics. The manifold of the reduced model is approximated by applying the so-called invariance condition together with repeated integrations of the reduced model in an iterative way. The latter is needed to improve the estimate of gradients of the reduced model parameters (coordinates which define the reduced manifold locally). To verify the approach onedimensional stationary laminar methane/air and syngas/air flames are investigated. In particular, it is shown that the adaptive REDIM method recovers the full stationary system dynamics governed by detailed chemical kinetics and the molecular transport in the case of a one dimensional reduced model and, therefore, includes the so-called flamelet method as a limiting case.
\end{abstract}

(C) 2009 The Combustion Institute. Published by Elsevier Inc. All rights reserved.

Keywords: ILDM; Reduction; Invariant manifolds; Laminar and diffusion flames

\section{Introduction}

Dimension reduction strategies for reacting flow systems are widely used in reacting flow calculations. Typically, detailed chemical kinetic models in engineering applications are extremely large, stiff and non-linear [1-3]. Even for small hydrocarbons, the number of species can easily exceed 100, and the number of reactions several thousands. As a result the detailed models can generally not be used for simulations of complex reacting flows in engineering problems. Although

\footnotetext{
* Corresponding author. Fax: +49 7216083931.

E-mail address: bykov@itt-uni-karlsruhe.de (V. Bykov).
}

the computational power and the efficiency of the numerical schemes have been improved constantly over the last decades there is a strong need for algorithms that handle the enormous dimension, stiffness, and non-linearity of reacting flow systems.

Therefore, methods performing automatic model reduction have to be devised. At present there are a lot of methods to reduce the stiffness, the dimension, and the CPU time and memory storage (see e.g. [4-7] for an overview of methods). Most of the existing methods exploit the so-called natural multi-scale structure of the system of governing equations. It is assumed that there are some fast modes or processes which are relaxed quickly and that only the slow processes govern 
the overall system dynamics. As a result, the long term system evolution is represented primarily by the dynamics of the slow reactions along a stable geometrical attractor with invariant properties, while the fast processes are relaxed. Moreover, removing the fast modes and reducing the dimension decreases the stiffness of the system of differential equations. This in turn allows larger time steps during the integration and, therefore, solves one of the major problems of the numerical implementation.

For that reason, most of the existing reduction methods try to characterize this decomposed structure and then use this special representation for reduction purposes. Of course this decomposition does not always exist and the assumption that the fast motions are not important for the overall system dynamics does not generally hold $[8,9]$. However, for reacting flow systems the validity of this approach is confirmed by the fact that the chemical kinetics system accesses only a small portion of the state or composition space [10].

Another difficult and fundamental problem of model reduction is the coupling of molecular transport with thermo-chemical processes. It is a very difficult problem normally solved by either neglecting such an interaction globally or by applying operator splitting methodologies (see e.g. [11]), which implies neglecting of the coupling locally either in time or in space. In both cases, the interaction is not taken into account and excluded from the reduced model. Hence, typically only the thermo-chemical source term is analyzed to yield the reduced model. There are a few exceptions like the so-called flamelet method $[12,13]$, which is based on detailed system solutions and, therefore, naturally accounts for this coupling (see e.g. [9] for more references and approaches).

In the present work, we further develop an approach (Reaction-Diffusion Manifold methods-REDIM $[14,15])$, which is able to treat the influence of the transport processes on the reduced model. In the previous work [14], the transport processes were included into the reduced description through an estimate of the local parameter gradient (of the low dimensional slow manifold which approximates the stationary system's dynamics), and the dependence on this estimation has been studied in detail. Now, by using an iterative procedure to solve an invariance equation $[7,14]$ and a test integration of the resulting reduced model, the problem of parameter's gradient estimation is overcome effectively. It is important to note, that the 1D REDIM method considered here, being used with exact gradients from flame simulations, fully corresponds to the flamelet method simply because it reproduces the detailed stationary solution. However, it allows an extension to higher dimensions and, therefore, permits to attack problems where 1D flamelets cease to describe the complex chemistry-transport interaction in an accurate way.

\section{Mathematical description}

In the following the mathematical concept of the REDIM method is presented together with the suggested improvement. In order to simplify the presentation of the suggested technique let us first introduce a vector notation. The vector $\psi=\left(\psi_{1}, \ldots, \psi_{n}\right)$ will characterize the thermochemical state of the system, where $\psi_{\mathrm{i}}$ represents thermo-chemical quantities as the pressure of mixture, the enthalpy, the mass fraction of chemical species etc. In this vector notation the system of governing equations for a reacting flow can be written as [14]

$$
\begin{aligned}
& \frac{\partial \psi}{\partial t}=\Phi(\psi) \equiv F(\psi)+G\left(\psi, \nabla \psi, \nabla^{2} \psi\right), \\
& \psi=\psi(x, t) \in R^{\mathrm{n}},
\end{aligned}
$$

where the first term is related to chemical kinetics (it is also called a source term) and the second one describes the physical processes (convection and diffusion):

$G\left(\psi, \nabla \psi, \nabla^{2} \psi\right)=-v \operatorname{grad} \psi-\frac{1}{\rho} \operatorname{div}(D \operatorname{grad} \psi)$.

Here $v$ is the flow velocity, $\rho$ - the density and $D$ is the generalized diffusion matrix [16]. Initial and boundary conditions depend on the structure of the considered problem, but e.g. for a premixed one dimensional flame, one boundary corresponds to the fresh combustible mixture composition, while the other is the completely reacted/burnt state behind the flame front. For non-premixed flames boundary conditions are, e.g., pure fuel or oxidizer, or (for two-dimensional REDIM) the mixing line between fuel and oxidizer and the curve of complete reaction to the products.

The assumption that an invariant (with respect to the system Eq. (1)) slow manifold $M_{\mathrm{s}}$ $\left(\operatorname{dim} M_{\mathrm{s}}=m\right)$ of low dimension exists in the state space:

$M_{\mathrm{s}}=\left\{\psi=\psi(\theta), \theta \in R^{\mathrm{m}}\right\}, \quad m \ll n$

leads to a straightforward application of the geometrical theory (see e.g. [7]) and yields the following equation for the manifold's explicit representation $\psi(\theta)$ in Eq. (3):

$\left(\psi_{\theta}\left(\theta_{0}\right)\right)^{\perp} \cdot \Phi\left(\psi\left(\theta_{0}\right)\right)=0$,

which means that the flow of the vector field Eq. (1) belongs to the tangent subspace of the manifold at any point $\theta_{0}$ on it. $\left(\psi_{\theta}\left(\theta_{0}\right)\right)^{\perp}$ denotes the orthogonal complement of the tangent space. An equivalent way to represent this condition, which 
actually has been used in the REDIM formulation, is given by (see [14] for more detail)

$\left(I-\psi_{\theta}\left(\theta_{0}\right) \psi_{\theta}^{+}\left(\theta_{0}\right)\right) \cdot \Phi\left(\psi\left(\theta_{0}\right)\right)=0$.

It states that the projection onto the normal subspace of the vector field vanishes on the slow invariant manifold. The Moore Penrose pseudoinverse matrix $\psi_{\theta}^{+}=\left(\psi_{\theta} \cdot \psi_{\theta}^{\mathrm{T}}\right)^{-1} \psi_{\theta}^{\mathrm{T}}$ (which always exists when the tangent space does not degenerate) and the identity matrix $I$ are applied to define the projection operator $P_{\mathrm{M}_{\mathrm{s}}^{\perp}}=\left(I-\psi_{\theta} \psi_{\theta}^{+}\right)$.

In order to solve Eq. (5) and approximate the manifold in a certain defined domain of the state space an iterative procedure has been suggested in [15] based on a reformulation as a multidimensional parabolic system:

$$
\left\{\begin{array}{l}
\frac{\partial \psi(\theta)}{\partial t}=\left(I-\psi_{\theta}(\theta) \psi_{\theta}^{+}(\theta)\right) \cdot \Phi(\psi(\theta)) \\
\psi^{0}=\psi^{\mathrm{ex}}(\theta)
\end{array}\right.
$$

with initial and boundary conditions given by an extended ILDM manifold $\psi=\psi^{\mathrm{ex}}(\theta)$, which has been introduced in [14], or by some other initial guess. The stationary solution of Eq. (6) satisfies exactly the invariance condition given by Eqs. (4), (5) (see e.g. [7]) and, therefore, approximates the manifold needed for the reduced system formulation. Now, the only problem with Eq. (6) is the transport term which, after substitution of Eq. (3) into Eq. (2) and after applying the equal diffusivity assumption $D=d \cdot I$, contains a term describing the dependence of the reduced model on the spatial variable (see [15] for more rigorous consideration). Note that an assumption of equal diffusivities is applied here for simplicity only; a generalization of the concept for non-equal diffusivities is possible without principle difficulties. Thus, Eq. (6) has the following simplified form: the dimension of the reduced model does not coincide with the spatial dimension of the considered problem. Even for equal dimensions singularities can occur perturbing a one-to-one correspondence between the parameter and its spatial gradient. In the current stage of the study such situations are excluded from consideration. Moreover, these problems could be easily overcome by using estimates for the components of missing dimensions in the $\operatorname{gradient} \operatorname{grad} \theta$. Furthermore, typically, the above mentioned singularities are a result of the high complexity of hydrodynamic structure of the combustion process and occur in transient regimes, which we are not focusing on in the current study.

\subsection{General algorithm}

Now, let us present the proposed method, which does not depend on the gradient estimates and, therefore, represents a better way to overcome the problem of dependence on the spatial coordinates. An additional iterative procedure is suggested by determining automatically the parameter gradient as a function of the parameter itself. A constant approximation $\operatorname{grad} \theta=c^{*}=$ const for this dependence in Eq. (7) is assumed in order to obtain the first approximation of the slow manifold $\psi^{1}(\theta)$. In [15] it has been shown that even for the constant approximation it is possible to achieve an appropriate accuracy of the reduced model, especially, if the dimension of the reduced model is reasonably high. Next, the reduced model $\psi^{1}(\theta)$ is used for integration to obtain the reduced model's stationary solution $\theta^{1}(x)$ by solving the following reduced system:

$\frac{\partial \theta(x, t)}{\partial t}=\psi_{\theta}^{1^{+}}(\theta(x, t)) \Phi\left(\psi^{1}(\theta(x, t))\right)$.

$$
\left\{\begin{array}{l}
\frac{\partial \psi(\theta)}{\partial t}=\left(I-\psi_{\theta}(\theta) \psi_{\theta}^{+}(\theta)\right) \cdot\left(F(\psi(\theta))-\frac{d}{\rho} \operatorname{grad} \theta \circ \psi_{\theta \theta}(\theta) \circ \operatorname{grad} \theta\right) . \\
\psi^{0}=\psi^{\mathrm{ex}}(\theta)
\end{array}\right.
$$

The convection term plays no role in the description of the invariant manifold, because it formally cancels out after applying the invariance condition Eq. (5) and rewriting it as the system Eq. (7) defining the manifold's evolution to the invariant form (see [15]). The dependence on the variables' spatial gradients was studied in detail in [15], where a constant approximation of the parameter's gradient $\operatorname{grad} \theta$ was introduced. Now, we suggest a method to approximate the reduced system gradient as a function $\operatorname{grad} \theta=f(\theta)$ of the parameter of the reduced manifold. Note, however, that in general this correspondence is not always well defined due to possibly differing dimensions: the spatial dimension of the problem and the reduced model dimension. This happens if
Then, $\theta^{1}(x)$ is used to find an approximation of the gradient of the manifold's parameter as $\operatorname{grad} \theta=\frac{\partial \theta^{1}(x)}{\partial x}$ and the gradient's dependence on $\theta$, $\operatorname{grad} \theta=f_{1}(\theta)$, is determined as follows: within the range of $\theta$ we define the spatial coordinate $x^{*}$ which corresponds to some fixed value of the parameter $\theta^{*}$, and in order to find the gradient at $\theta^{*}$, we calculate the derivative at this spatial point:

$\theta_{*}=\theta^{1}\left(x_{*}\right) \quad \Rightarrow \quad f_{1}\left(\theta_{*}\right)=\left.\frac{\partial \theta^{1}(x)}{\partial x}\right|_{x=x_{*}}$.

The rest is obvious; the better representation of the gradient on the manifold is applied to improve the reduced manifold by using $f_{1}(\theta)$ in Eq. (7) 
together with $\psi^{0}=\psi^{1}(\theta)$ as an initial guess. After the relaxed (iterated until convergence) solution of Eq. (7), $\psi^{2}(\theta)$, has been found, it can be used in Eq. (8) to update the gradient dependence $\operatorname{grad} \theta=f_{2}(\theta)$, etc. Note that Eq. (7) represents a continuous version of the invariance condition given by Eq. (5), such that the stationary solution of Eq. (7), defining a manifold satisfies exactly the invariance condition, whereas Eq. (8) is the governing equation for the reduced state space specified as a confinement of the system Eq. (1) to the invariant manifold Eq. (7).

The main steps of the REDIM adaptation concept and the detailed implementation scheme are given as follows:

(1) Generate an extended ILDM $\psi^{\mathrm{ex}}(\theta)$, $\theta=\left(\theta_{1}, \ldots, \theta_{m}\right)$ as an initial guess (see [14] for details).

(2) Estimate the initial guess of the parameter's gradient $\operatorname{grad} \theta=f_{0}(\theta)=$ const.

(3) Integrate Eq. (7) to find the stationary solution $\psi=\psi^{1}(\theta)$.

(4) Integrate Eq. (8) and improve the gradient dependence $\operatorname{grad} \theta=f_{1}(\theta)$ based on the stationary solution Eq. (9).

(5) Return to step (3) and repeat iteration until convergence is reached.

The REDIM method has been implemented in the codes INSFLA and HOMREA [10,17]. It has to be noted that there are some crucial theoretical issues like, for instance: the convergence of the suggested iterative procedures; a rigorous definition of the dependence grad $\theta=f(\theta)$ in case of differing dimensions of spatial and reduced spaces; the existence of the slow manifold in the state space etc., which will be analyzed and studied in the near future. For example, one of main obstacles with the application of the method is in the consistent definition of boundary conditions for Eq. (7). One possible way to overcome the problem (for arbitrary initial guess of the invariant manifold) can be a hierarchical implementation of the method that applies as a boundary an invariant manifold of lower dimension. The problem with different dimensions of the spatial and reduced spaces, which is in the list above, can be handled similarly as it has been done here for the case of equal dimensions. Namely, one can use iteratively the reduced model stationary solutions, in order to improve not the exact gradients, but the gradient's estimate. Note that with the increase of the reduced model dimension (see [15] for more details and discussions) the dependence of the reduced model on the gradient's estimation becomes weaker.

In order to apply the method to an unsteady problem describing transient regimes of combustion, one can follow the standard procedure of the ILDM or flamelet methods, namely, one can use at any time step of integration an appropriate table defining the reduced model (assuming the solution has already relaxed onto a lower dimensional manifold). Instationary problems can also be described by low-dimensional manifolds, although maybe the required dimension might be higher. Due to the general formulation for arbitrary dimensions the method is able to handle such problems. In the following different structures of laminar flames are used to validate the model. Stability and robustness of the method are demonstrated, convergence, however, is tested a posteriori.

\subsection{Illustrative example: Michael Davis and Rex Skodje model}

As an illustration of the suggested iterative approach let us take the well known example of Davis-Skodje often used as a test/benchmark case (see e.g. [18-20]):

$$
\left\{\begin{array}{l}
\frac{\partial y_{1}}{\partial t}=-y_{1}+D \frac{\partial^{2} y_{1}}{\partial x^{2}}, \quad x \in[0,1] \\
\frac{\partial y_{2}}{\partial t}=-\gamma y_{2}+\frac{(\gamma-1) y_{1}+\gamma y_{1}^{2}}{\left(1+y_{1}^{2}\right)}+D \frac{\partial^{2} y_{2}}{\partial x^{2}}
\end{array},\right.
$$

with the following boundary and initial conditions

$$
\begin{aligned}
& \left(\begin{array}{l}
y_{1}(t, 0) \\
y_{2}(t, 0)
\end{array}\right)=\left(\begin{array}{l}
0 \\
0
\end{array}\right), \quad\left(\begin{array}{l}
y_{1}(t, 1) \\
y_{2}(t, 1)
\end{array}\right)=\left(\begin{array}{l}
1 \\
3 / 4
\end{array}\right), \\
& \left(\begin{array}{l}
y_{1}(0, x) \\
y_{2}(0, x)
\end{array}\right)=\left(\begin{array}{l}
x \\
3 x / 4
\end{array}\right) .
\end{aligned}
$$

The commonly used system parameters are $\gamma=10,100 ; D=0.1$, where $\gamma$ is the large system parameter, which defines the differences in chemical time scales and, consequently, the stiffness of the system's source term. In the two dimensional case only a one-dimensional reduced model exists, therefore, the reduced manifold has to coincide with the system's stationary solution. In this case it is very simple to control and compare the detailed and reduced models generated by the improved REDIM method. In this example a straight line connecting boundary values in the state space $\left(y_{1}, y_{2}\right)$ is used as an initial guess for all approximations of the invariant manifold $M_{\mathrm{s}}^{\text {in }}=\left\{\left(y_{1}^{\text {in }}(\theta), y_{2}^{\text {in }}(\theta)\right), \theta=1, \ldots, N\right\}$. Then by solving Eq. (7) the first approximation $M_{\mathrm{s}}^{1}=\left\{\left(y_{1}^{1}(\theta), y_{2}^{1}(\theta)\right), \theta=1, \ldots, N\right\} \quad$ is obtained. This solution is used to solve Eq. (8) yielding the stationary solution $\theta=\theta^{1}(x)$, which is then used to define $\operatorname{grad} \theta=f_{1}(\theta)$ for the next iteration step.

In Fig. 1 the influence of the parameter's gradient is studied. The number of points for both the manifold's parameterization and the spatial variable is chosen to be $N=100$. The first approximation deviates significantly from the initial guess (compare straight dotted line and first iteration in Fig. 1 on the left). Because the gradient is underes- 

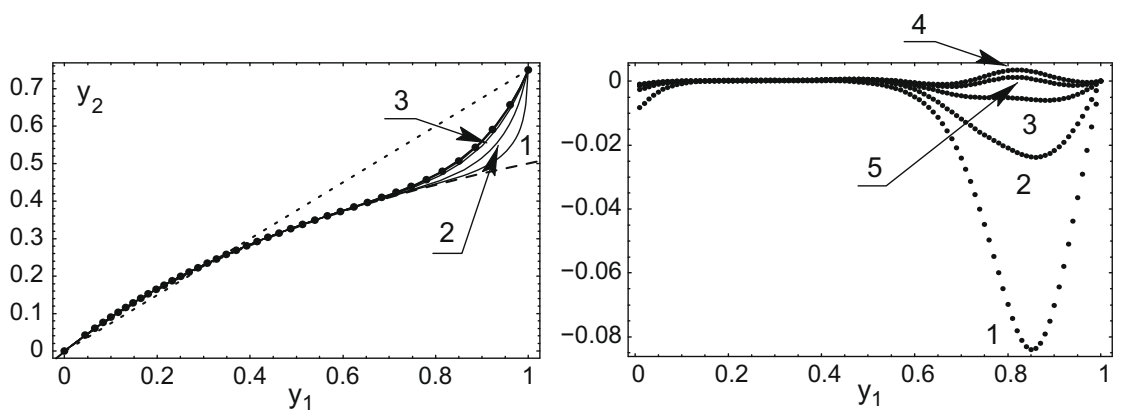

Fig. 1. Iteration process for $\operatorname{grad} \theta=1, N=100$. The left figure shows the first 5 iterations of the slow manifold's approximation (solid lines) and an exact solution (filled circles). The dotted line shows the initial guess of the invariant curve and the dashed line corresponds to the invariant manifold based on the source term only, the right figure represents the relative error of the iterations.

timated, the reduced curve is governed mainly by the source term. It is known that the source term of this model yields the invariant slow manifold given by $y_{2}=y_{1} /\left(1+y_{1}\right)$ (see e.g. [21] and Fig. 1 dashed line), which attracts the solution of Eq. (7) if the transport term is small. During the next iterations the solution quickly approaches the stationary system solution. After the third iteration, it is not possible to distinguish further solutions in Fig. 1 left. The considered example illustrates that the method gives an impression of the general behavior of the iterations and can be useful to enhance better understanding of the behavior of more complex combustion models in the state space.

\section{Results: syngas and methane/air laminar flames}

To verify the approach in detail one-dimensional stationary premixed and diffusion flames have been simulated by using both detailed and reduced chemical kinetic models. The detailed description of mathematical models that have been used below for illustration purposes can be found in $[1,10,17]$.

\subsection{Premixed flame, methanelair stoichiometric system}

At first, the methane/air combustion system $(n=36)$ has been examined in the laminar premixed combustion regime to validate the onedimensional reduced model $(m=1)$. The results are summarized in Fig. 2 which shows specific mole numbers of some major and minor species. The initial gradient in this case is estimated by the following empirical function. It merely acts as a starting guess,

$C(x)=\exp \left(-\frac{2(x-a)^{\mathrm{b}}}{a}\right)$, where $b=8, a=0.01 \mathrm{~m}$ have been chosen to represent the typical flame thickness. Note, however, that an arbitrary choice of the estimate is also possible, but would probably need more iterations to yield the fully converged solution. Then, the initial or starting solution yielding an initial guess for the reduced manifold in the state space is defined by the straight line joining the mixing/unburnt $\psi^{\mathrm{u}}$ and equilibrium/burnt $\psi^{\mathrm{b}}$ states

$\psi_{\mathrm{i}}(x, 0)=(1-C(x)) \psi_{\mathrm{i}}^{\mathrm{u}}+C(x) \psi_{\mathrm{i}}^{\mathrm{b}}, \quad i=1, \ldots, n$.

It can be interpreted as an initial guess of the manifold (can be seen in Fig. 2) instead of the ILDM used in definition of Eq. (7). Then we find the stationary solution of Eq. (7). The generalized coordinate $\theta$ is applied to parameterize the straight line Eq. (13) (see e.g. $[14,16]$ ) and, therefore, the parameter's gradient:

$$
\begin{aligned}
& \operatorname{grad} \psi_{\mathrm{i}}(x, 0)=\left(1-C^{\prime}(x)\right) \psi_{\mathrm{i}}^{\mathrm{u}}+C^{\prime}(x) \psi_{\mathrm{i}}^{\mathrm{b}}, \\
& i=1, \ldots, n \operatorname{grad} \theta=\psi_{\theta}^{+} \operatorname{grad} \psi=f_{0}(\theta) .
\end{aligned}
$$

Now let us show how the REDIM method is implemented. First, Eq. (7) is solved with the initial guess given by Eq. (13) and gradient estimated using Eq. (14) to yield $\psi^{1}(\theta)$. Next Eq. (8) is integrated to improve the gradient dependence on the parameter $\theta$ Eq. (14) giving $\operatorname{grad} \theta=f_{1}(\theta)$, etc. (see Fig. 2).

In Fig. 2 on the right, the initial guess can not be seen because it is very close to the $x$ axis. In particular, one can see that starting with the straight line (not even an extended ILDM!) the iteration converges fast and we do not notice any changes in the manifold after the third iteration. To see how the reduced model defines the properties of the reacting flow we calculate the relative error (with respect to the detailed solution) of the stationary flame velocities. In the considered example the relative errors are $28.3 \%, 3.4 \%$, $0.8 \%$ and decrease with the following error dependence on the iterations $\exp [-1.77 i], i=1,2,3$, 

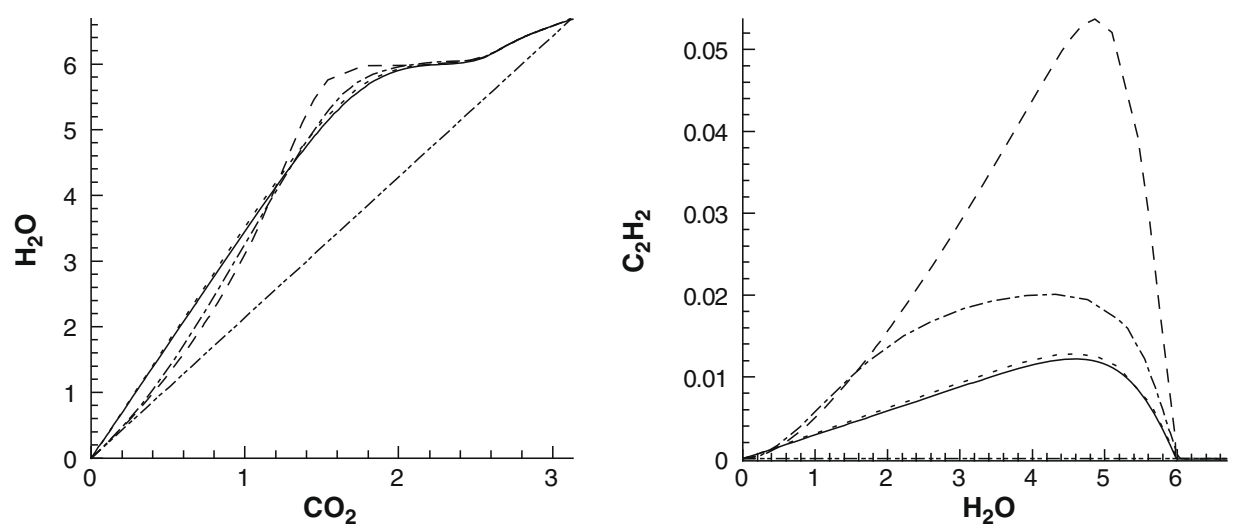

Fig. 2. Laminar premixed methane/air flame. $\mathrm{CO}_{2}-\mathrm{H}_{2} \mathrm{O}$ plane (left) and $\mathrm{H}_{2} \mathrm{O}-\mathrm{C}_{2} \mathrm{H}_{2}$ plane (right) projections of the state space with stationary solutions (specific mole numbers). Solid line is detailed stationary solution, dotted line: initial guess according to Eq. (11), dashed line: result of the first iteration, the dash-dot line - second and dash-dot-dot line the third one.

which means that the relative error decreases exponentially for the considered system parameters.

\subsection{Premixed flame, syngas/air stoichiometric system}

The second combustion model is a premixed flame of carbon monoxide/hydrogen/nitrogen/ oxygen $\left(\mathrm{CO} / \mathrm{H}_{2} / \mathrm{N}_{2} / \mathrm{O}_{2}\right)$, the so-called syngas/air system. In this case the overall system dimension is 15 and the system dimension, now, is reduced to two dimensions $(m=2)$. A $2 \mathrm{D}$ reduced model is compared to the extended ILDM [14], and the detailed solution. The gradients of the parameter of the REDIM reduced model are estimated on a basis of the extended ILDM, exactly as it has been described in Section 2. Already the first step of the implementation scheme gives a quite accurate REDIM manifold, which does not change notably in further iterations. The flame velocity estimated by the 2D REDIM model is within one percent accuracy in comparison to the detailed one. The profiles are presented and compared in Fig. 3(a-c). The comparison between the stationary solutions shows that in the domain close to states corresponding to the fresh mixture, the REDIM improves considerably the flame structure not only for the major, but for minor species as well (c and d). Note that in large parts of the domain the adaptive REDIM solution cannot be distinguished from the detailed solution.

\subsection{Diffusion flame, syngas combustion system}

The third test case is a diffusion flame of the syngas/air system described above. The starting linear solution in the state space for the diffusion flame joins the points corresponding to the fresh mixture of the fuel mixture flow from one side with the point describing the air flow from the other side. The REDIM iteration procedure, Eqs. (7) and (8), has been performed with an initial estimation of the local parameter gradient given by $\lambda(\operatorname{grad} \theta)^{2} / \rho c_{\mathrm{p}}=5 \times 10^{3}$ (see e.g. [14]). The estimate has been chosen to agree with the strain rate of the counter-flow and with the detailed solution within the same order of magnitude.

Figure 4 shows that already the second iteration matches well the detailed solution in the state space projection for minor species like $\mathrm{CH}_{2} \mathrm{O}$, and also reproduces accurately the system profile $(\mathrm{OH})$ in spatial coordinates. Of course the first iteration fails to give an appropriate solution because it was based on the rough constant estimate of the parameter gradient.

\section{Conclusions}

An iterative method for determining an approximation of an invariant manifold of slow motions in the state space for complex combustion problem has been presented and discussed. It is based on the so-called invariance equation and a set of reduced model solutions. The manifold is approximated by a mesh representing a low-dimensional manifold in the composition or state space. The mesh is tabulated by vertices having integer indices and used as the generalized coordinates. The invariance equation is solved iteratively to yield the approximation of the manifold, while the reduced model is integrated in order to obtain an improved parameter's gradient as a function of the parameter (local coordinate of the manifold). The procedure is recursive and allows improving the reduced model step by step. 

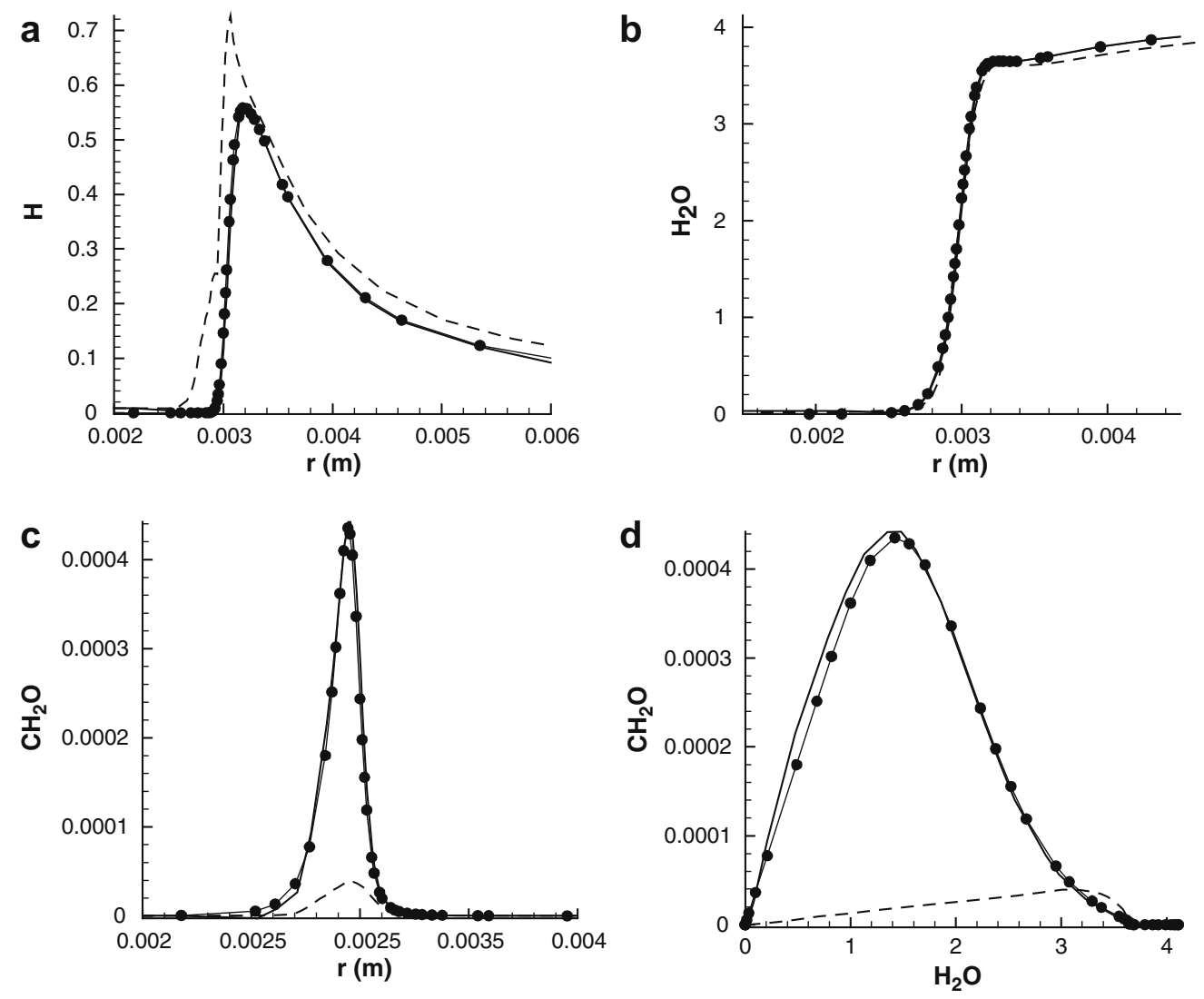

Fig. 3. Laminar premixed syngas/air flame. $\mathrm{H}, \mathrm{H}_{2} \mathrm{O}, \mathrm{CH}_{2} \mathrm{O}$-profiles (a, b, c) and projection of the state space with the stationary solution onto $\mathrm{H}_{2} \mathrm{O}-\mathrm{CH}_{2} \mathrm{O}$ plane (specific mole numbers, d). The detailed solution is shown by the solid line with filled circles, the dashed line represents the result of the 2D extended ILDM, the solid line is the result of the 2D REDIM model.
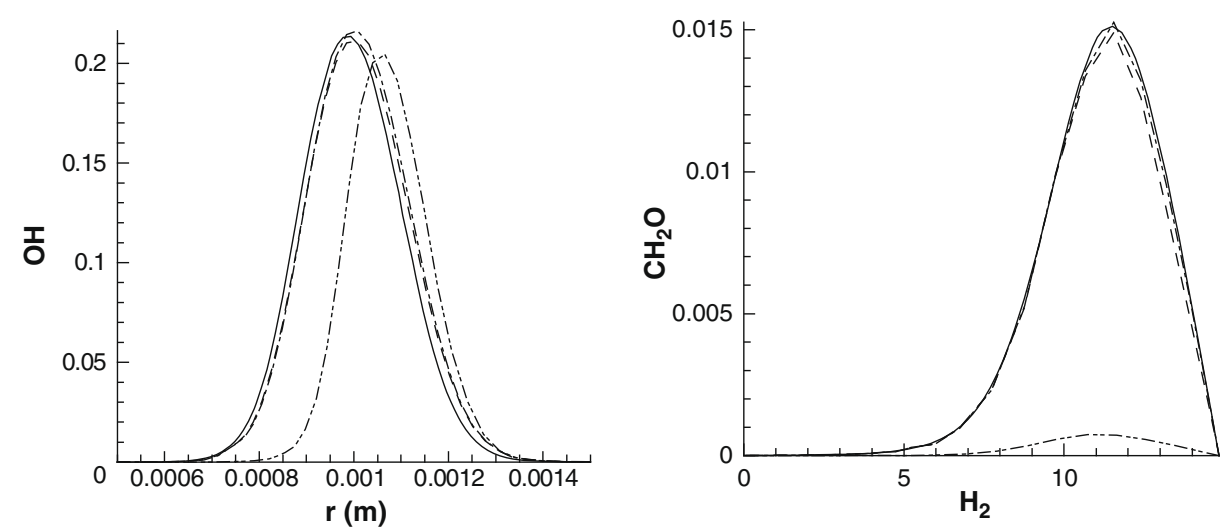

Fig. 4. Diffusion flame. OH-profile (left) and projection of the state space with the stationary solution onto $\mathrm{H}_{2}-\mathrm{CH}_{2} \mathrm{O}$ plane (specific mole numbers, right). The detailed solution is shown by the solid line, the dotted line represents the result of the first iteration for the constant gradient over the spatial domain; the dashed line is the result of the second iteration and the dash-dot line corresponds to the third one.

The method is implemented in the standard ILDM code and applied to a number of meaningful test problems: premixed and non-premixed laminar flames. The results for both types of laminar flames show extremely fast convergence of the REDIM manifold to the stationary solutions 
representing the approximation of slow invariant system manifold. Although results have been shown for one-dimensional flames only, the method can also be used for more complex flows without principle difficulties.

\section{Acknowledgments}

This research was supported by the Deutsche Forschungsgemeinschaft (DFG) within the Sonderforschungsbereich (SFB 606).

\section{References}

[1] J. Warnatz, U. Maas, R.W. Dibble, Combustion, Springer Verlag, Berlin, 2000.

[2] N. Peters, B. Rogg, Reduced Kinetics Mechanisms for Application in Combustion Systems, Springer, Berlin, 1993.

[3] V. Warth, F. Battin-Leclerc, R. Fournet, P.A. Glaude, G.M. Côme, G. Scacchi, Comput. Chem. 24 (5) (2000) 541-560.

[4] J.F. Griffiths, Prog. Energy Combust. Sci. 21 (1995) 25-107.

[5] A.S. Tomlin, T. Tur'anyi, M.J. Pilling, Mathematical Tools for the Construction, Investigation and Reduction of Combustion Mechanisms, Comprehensive Chemical Kinetics 35: Low-temperature Combustion and Autoignition, Elsevier, Amsterdam, 1997.
[6] M.S. Okino, M.L. Mavrovouniotis, Chem. Rev. 98 (2) (1998) 391-408.

[7] A.N. Gorban, I.V. Karlin, A.Y. Zinovyev, Phys. Rep. 396 (2004) 197-403.

[8] V. Bykov, V. Gol'dshtein, U. Maas, Combust. Theory Model 11 (6) (2007) 839-862.

[9] S.H. Lam, Combust. Sci. Tech. 179 (2006) 767-786.

[10] U. Maas, Automatische Reduktion von Reaktionsmechanismen zur Simulation reaktiver Stroemungen. Habilitation thesis, Institut fuer Technische Verbrennung, Universitaet Stuttgart, Germany, 1993.

[11] M.A. Singer, S.B. Pope, H.N. Najm, Combust. Theory Model. 10 (2) (2006) 199-217.

[12] L.P.H. De Goey, J.H.M. Ten Thije Boonkkamp, Combust. Flame 119 (1999) 253-271.

[13] N. Peters, Proc. Combust. Inst. 21 (1987) 1231-1250.

[14] V. Bykov, U. Maas, Proc. Combust. Inst. 31 (2007) 465-472.

[15] V. Bykov, U. Maas, Combust. Theory Model. 11 (6) (2007) 839-862.

[16] J. Bauer, V. Bykov, U. Maas, Implementation of ILDMs based on a representation in generalized coordinates, Proceedings of the European Conference on Computational Fluid Dynamics, ECCOMAS CFD, Egmond aan Zee (The Netherlands), 2006.

[17] U. Maas, J. Warnatz, Combust. Flame 74 (1988) 5369.

[18] M.J. Davis, J. Phys. Chem. A 110 (2006) 5235-5256.

[19] M.J. Davis, J. Phys. Chem. A 110 (2006) 5257-5272.

[20] Z. Ren, S.B. Pope, Combust. Theory Model. 11 (5) (2007) 715-739.

[21] M.J. Davis, R.T. Skodje, J. Chem. Phys. 111 (1999) 859-874. 